\section{Passively mode-locked fiber ring laser with tunable repetition rate output}

\author{
Yandong Gong \\ Institute for Infocomm Research \\ Innovation Center \\ 18 Nanyang Drive \\ Singapore 637723 \\ E-mail: gongyd@i2r.a-star.edu.sg
}

\section{Ping Shum}

Nanyang Technological University

Network Technology Research Center

School of Electrical \& Electronic

Engineering

Singapore 639798

Chao Lu, MEMBER SPIE

Institute for Infocomm Research

Innovation Center

18 Nanyang Drive

Singapore 637723

\begin{abstract}
We propose and experimentally demonstrate a novel and simple tunable passively mode-locked fiber ring laser. A polarization-dependent Mach-Zehnder amplitude modulator is used to replace the polarizer inside the cavity. When the modulator is biased at different voltages and without any rf input signal, we obtain a stable soliton pulse train with repetition rates from 50 to $300 \mathrm{GHz}$. (C) 2005 Society of Photo-Optical Instrumentation Engineers. [DOI: 10.1117/1.1920527]
\end{abstract}

Subject terms: fiber lasers; pulses; erbium lasers; nonlinear optics.

Paper 050139 LR received Feb. 28, 2005; revised manuscript received Mar. 27, 2005; accepted for publication Mar. 29, 2005; appeared online Mar. 31, 2005; published online Jun. 14, 2005.

\section{Introduction}

To meet the rapidly expanding demand for high-speed communication systems, an ultra-high-speed soliton light source is indispensable not only for the development of ultra-high-speed optical time division multiplexing (OTDM) but also for future ultra-high-speed dense wavelength division multiplexing (DWDM) communications. ${ }^{1}$ Actively mode-locked fiber ring lasers have been shown to be an attractively inexpensive all-optical light source to generate high-speed, ultrashort pulse trains up to $40 \mathrm{GHz}$. Due to the limit of the "electronics neck," it is difficult to increase the repetition rate further. Usually passively modelocked fiber lasers can generate very narrow femtosecond pulses but with very low repetition rates on the order of megahertz. ${ }^{2}$ This frequency cannot be varied if the cavity is not redesigned. But any change in cavity will require a very long time to readjust the polarization in order to realize mode-locking in passively mode-locked fiber lasers. Bound soliton phenomena were first observed by our group in a

0091-3286/2005/\$22.00 @ 2005 SPIE

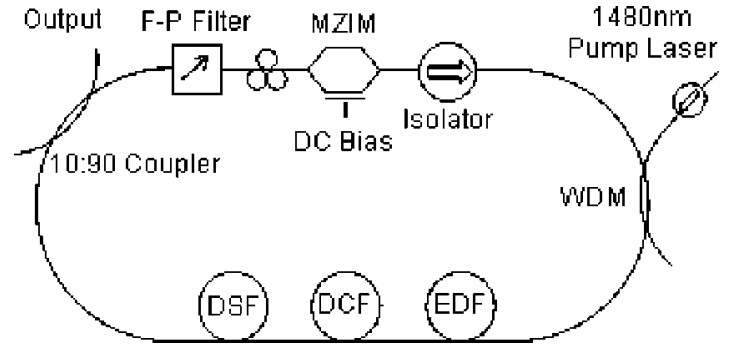

E

Fig. 1 Configuration of modified mode-locked fiber ring laser.

passively mode-locked fiber ring laser ${ }^{3}$ and we were able to increase the repetition rate. We also presented ${ }^{4}$ that 660 $\mathrm{GHz}$ soliton pulse trains were observed in a modified passively mode-locked fiber ring laser, which was based on a complex four-wave mixing (FWM) process. ${ }^{5}$ Owing to the simplicity of its configuration, the exploitation of FWM lasers has attracted growing attention.

The demerit of it is that its repetition rate can not be varied. Here we present that in a modified passively modelocked fiber ring laser, we used a M-Z amplitude modulator to replace the polarizer inside the cavity. Without any rf signal input, when the bias voltage was set at different voltages, the repetition rate of the output pulses changed accordingly, and we obtained a minimum of $50 \mathrm{GHz}$ and up to a $300-\mathrm{GHz}$ stable pulse train.

\section{Experimental Configuration and Results}

Figure 1 shows the configuration of the actively modelocked fiber ring laser. The cavity length is around $76 \mathrm{~m}$, which comprises an 8-m-long erbium-doped fiber (Er+ concentration $\sim 2000 \mathrm{ppm}$ ). A pigtailed 1480-nm laser diode is used to pump the laser. Unidirectional operation of the laser is enforced by the inclusion of a polarizationindependent isolator in the cavity. A Fabry-Perot (F-P) filter with a 3-dB bandwidth of $5.8 \mathrm{~nm}$ is inserted in the cavity to control the wavelength. A 40-GHz bandwidth $\mathrm{LiNbO}_{3} \mathrm{M}-\mathrm{Z}$ amplitude modulator was inserted into the cavity to replace the polarizer. Due to its polarization dependence, we can utilize it as a tunable 'polarizer.' From the modulator's transfer curve, it is well known that when we adjusted the bias voltage, its transmittivity and the extinction ratio of this 'polarizer' was changed accordingly. 50-m dispersion-

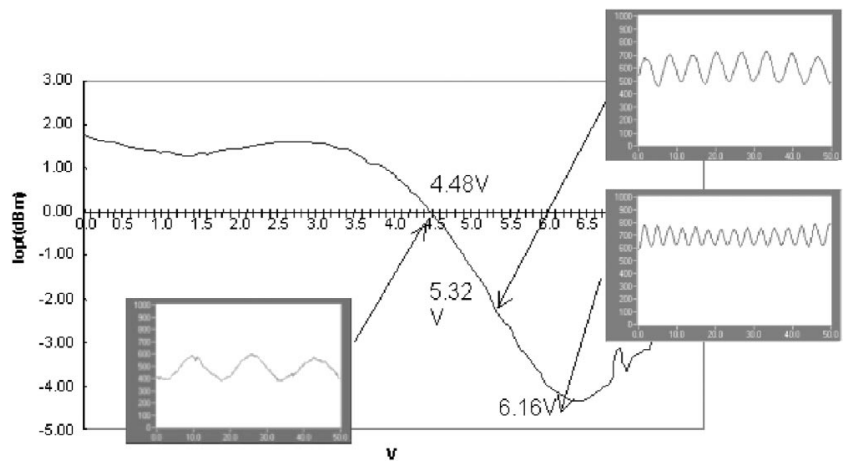

Fig. 2 Output variation of mode-locked pulses along modulator transfer curve. 


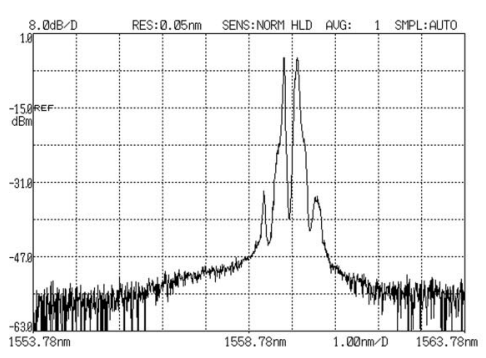

(a) $4.48 \mathrm{~V}$

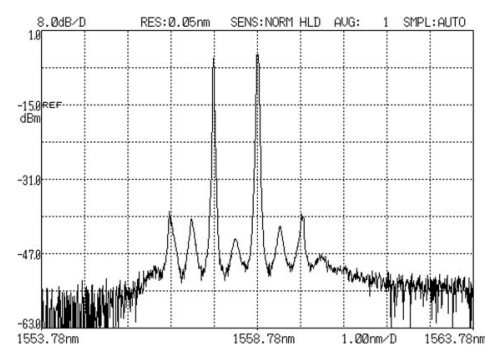

(b) $5.32 \mathrm{~V}$

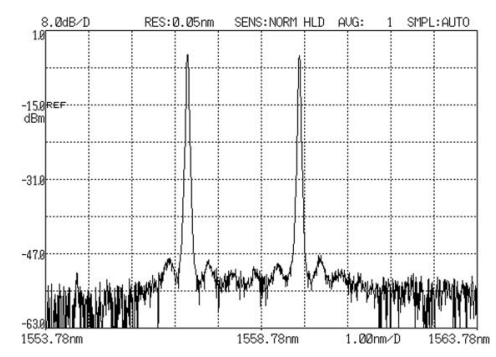

(c) $6.16 \mathrm{~V}$

Fig. 3 Optical spectrums at different dc bias voltages.

shifted fiber (DSF) and 1.1-m dispersion compensation fiber (DCF) were added to increase nonlinearity and to compensate the cavity dispersion, respectively.

The laser pump power was kept high by setting the pump current at $600 \mathrm{~mA}$ or above, which is equivalent to $120 \mathrm{~mW}$ approximately. By carefully adjusting the polarization controller, mode locking can be realized and a highspeed soliton train can be generated. When the different dc bias voltage of the modulator was set, the pulses would transform to be of different mode-locked frequencies. Hence, the ring laser was described as tunable. The modelocked frequencies obtained were around 50, 130, and 300 $\mathrm{GHz}$. There are even narrow continuously tunable ranges for each repetition rate, respectively, for example, from 45 to $60 \mathrm{GHz}, 100$ to $160 \mathrm{GHz}$, and 280 to $310 \mathrm{GHz}$ and so on. Some results are displayed in Fig. 2, which is the modulator transfer curve; the insets give the outputs of the autocorrelator and their corresponding bias voltages. Figures 3(a) to 3(c) illustrate the corresponding optical spectrum of Fig. 2 during mode-locked situations, with repetition rates at 50, 130, and $300 \mathrm{GHz}$, respectively. At each fixed condition, it can keep stable output for several minutes. If the hyperbolic pulse profiles are assumed, their pulsewidths are $7.8,3.2$, and $1.4 \mathrm{ps}$, respectively, and then the product of time and bandwidth are all less than 0.4 , which means the output pulses are all almost Fourier transform limited.

The mechanism of producing such high-repetition-rate pulse trains and simulations has been presented by Thibaut, ${ }^{5}$ based on the complex-FWM process. It is well known that dispersion influence is very obvious in the FWM effect; it decides the phase matching and frequency. The reason why our laser is tunable is that the bias changes the modulator's refractive index, leading to a different optical path in the cavity, which in turn leads to different dispersion. This will change the value of the dispersion parameter. Thus, different repetition rates are generated. When working at other regions, for example, the flat transfer curve regime where the bias is below $3.0 \mathrm{~V}$, we also attained different high-speed output pulses.

\section{Conclusions}

A passively complex-FWM mode-locked fiber soliton ring laser was established in which an amplitude modulator without rf signal input was used to replace the polarizer inside the cavity. Its output repetition rate can be tuned. When the bias voltage of the modulator was adjusted, its transmissibility was changed accordingly, which may lead to the change of refractive index and dispersion, so that a stable and tunable rate from 50 to $300 \mathrm{GHz}$ is obtained.

\section{References}

1. A. D. Kim, J. N. Kutz, and D. J. Muraki, "Pulse-train uniformity in optical fiber lasers passively mode locked by nonlinear polarization rotation," IEEE J. Quantum Electron. 36(4), 465-471 (2000).

2. J. M. Dudley, L. P. Barry, J. D. Harvey et al., "Complete characterization of ultrashort pulse sources at $1550 \mathrm{~nm}$," IEEE J. Quantum Electron. 35(4), 441-450 (1999).

3. Y. D. Gong, P. Shum, D. Y. Tang, and T. H. Cheng, "Bound soliton pulses with FWHM duration of $326 \mathrm{fs}$ and separation of $938 \mathrm{fs}$ in a passively mode locked fiber laser," OFC'2002 Proc., Anaheim, California, ThGG, pp. 655-656 (2002).

4. Y. D. Gong, P. Shum, D. Y. Tang, and C. Lu, " $660 \mathrm{GHz}$ passively mode-locked soliton fiber laser at $1566 \mathrm{~nm}$ wavelength," European Conference on Optical Communication-International Conference on Integrated Optics and Optical Fibre Communication Proc., Rimina, Italy, Vol. 3, We4.P.3, pp. 562-563 (2003).

5. T. Sylvestre, S. Coen, P. Emplit, and M. Haelterman, "Self-induced modulational instability laser revisited: normal diserpsion and darkpulse train generation," Opt. Lett. 27(7), 482-484 (2002). 